\title{
Exploring factors in fear of COVID-19 and its GIS-based nationwide distribution: the case of Bangladesh
}

\author{
Mohammed A. Mamun
}

\section{Background}

The COVID-19 pandemic is a public health threat of international concern, intensifying peoples' psychological risk and vulnerability by strengthening mental health stressors such as fear panic and uncertainty. The unexpected fear of COVID-19 has been reported to be associated with suicide occurrences, similar to prior pandemics.

\begin{abstract}
Aims
Identifying the factors associated with fear of COVID-19 could help us to develop better mental health strategy and practice to improve the situation here in Bangladesh. This was the first attempt to present a Geographic Information System (GIS)-based distribution of fear of COVID-19 across the country's administrative districts in a nationwide sample.

\section{Method}

Data for a total of 10067 individuals were collected by an online survey during the first wave of the pandemic (1 to 10 April 2020); data for 10052 participants were finally analysed after excluding 15 transgender individuals. The survey questionnaire included items concerning sociodemographic, behavioural and healthrelated variables, COVID-19-related issues, and the Bangla Fear of COVID-19 Scale.
\end{abstract}

\section{Results}

The mean fear of COVID-19 scores was $21.30 \pm 6.01$ (out of a possible 35 ) in the present sample. Female gender, highly educated, non-smoker, non-alcohol consumer, having chronic diseases, using social media, and using social media and not using newspapers as COVID-19 information sources were associated with a higher level of fear of COVID-19.
Higher levels of fear of COVID-19 were found in districts of Magura, Panchagarh, Tangail, Sunamganj and Munshiganj; by contrast, Kushtia, Pirojpur, Chapainawabganj, Jhalokathi and Naogaon districts had lower fear of COVID-19. Based on the GISdistribution, fear of COVID-19 was significantly associated with the district as well as in respect to its gender-based and education-level-based associations. However, fear of COVID-19 and COVID-19 cases were heterogeneously distributed across the districts; that is, no consistent association of higher COVID-19 cases with higher fear of COVID-19 was found.

\section{Conclusions}

This study being exploratory in nature may help to facilitate further studies, as well as directing governmental initiatives for reducing fear of COVID-19 in at-risk individuals. Providing adequate resources and mental health services in the administrative regions identified as highly vulnerable to fear of COVID-19 is recommended.

\section{Keywords}

COVID-19 fear; psychological impact; fear of infection; fear of COVID-19 scale; GIS-based distribution; Bangladesh.

\section{Copyright and usage}

(C) The Author(s), 2021. Published by Cambridge University Press on behalf of the Royal College of Psychiatrists. This is an Open Access article, distributed under the terms of the Creative Commons Attribution licence (http://creativecommons.org/ licenses/by/4.0/), which permits unrestricted re-use, distribution, and reproduction in any medium, provided the original work is properly cited.

\section{Introduction}

During viral outbreaks such as the COVID-19 pandemic, the perceived risk of infection with the virus increases emotional issues including fear, panic, worries and uncertainties. ${ }^{1}$ The effects of fear of COVID-19 on psychological health were visualised during the early period of the pandemic in Bangladesh. ${ }^{2}$ The first COVID-19-related suicide case occurred on 25 March 2020, with an allegation of fear of COVID-19 as the suicide stressor; ${ }^{3}$ whereas the first COVID-19 patient was diagnosed on 8 March 2020. ${ }^{4}$ Extreme psychological effects (such as suicide) related to fear of COVID-19 have not only been observed in Bangladesh; several case-series studies from other countries have also been reported. ${ }^{5,6}$ Similarly, fear of infection was regarded as a prominent suicide factor in prior pandemics. ${ }^{7,8}$ Studies have confirmed that fear of COVID-19 acts as a mediator to increase psychological events such as depression and anxiety, ${ }^{2}$ thereby making individuals psychologically vulnerable to suicidality. ${ }^{9}$ Thus, increments in suicide rates have been seen owing to burdens related to fear of COVID-19, as well as other devastating effects of the pandemic (see Mamun ${ }^{9}$ a systematic review of studies on suicide in Bangladesh),
For understanding the effects of fear of COVID-19 on people and adopting effective mental health strategies, there is a need for epidemiological studies. ${ }^{10,11}$ However, there has been a lack of studies assessing fear of COVID-19 compared with other aspects of mental suffering, including depression, anxiety and stress, in Bangladesh. ${ }^{11}$ To the best of the author's knowledge, three studies have assessed risk factors for fear of COVID-19 across different cohorts (i.e., the general population, ${ }^{12}$ the general population and healthcare professionals, ${ }^{4}$ and older adults ${ }^{13}$ ), whereas another study explored its mediating role in career anxiety among students. ${ }^{2}$ However, none of these studies considered such a large sample as that used in the present study, provided any predictive models explaining the fear of COVID-19, or considered its nationwide spatial distribution.

More engagement with COVID-19-preventive behaviours is frequently observed in individuals with higher COVID-19 fear, ${ }^{14}$ where fear of COVID-19 acts as a motivational factor. ${ }^{15}$ Whereas, practice of behaviours related to COVID-19 knowledge, fear and so on, increase among female individuals and those with higher education levels. ${ }^{16-18}$ However, none of the aforementioned Bangladeshi studies considered the associations of education level or gender with fear of COVID-19; these associations are investigated herein. 


\section{Method}

\section{Study procedure, participants and ethics}

The present nationwide cross-sectional study was conducted during the early stage of the COVID-19 pandemic, between 1 and 10 April 2020. The survey was conducted via online promotion; three or four research assistants from each of the 64 districts in Bangladesh were employed to facilitate access to the survey link through their social media platforms. The research team approached nearly 11000 participants; 10067 were enrolled in the present study after meeting the criteria of being Bangladeshi residents aged over ten years. Ethical approval for this project was obtained from two Bangladeshi institutes (see Ethics statement). For detailed information about the study sample, procedures, participants, ethics and data access, please refer to the following publications. ${ }^{19-23}$

\section{Measures}

Independent variable

Information related to basic sociodemographic variables including age, gender, education level, occupation status, marital status, current residence type and district of residence was collected in this study. Education level was categorised into two types: lower education (up to secondary high school) and higher education (tertiary education). Behaviour- and health-related variables (smoking status, perceived health status [based on a Likert scale], social media use, etc.) were also included in this study. Participants were asked whether anyone had returned to their home from Dhaka or outside the Bangladesh area/country which were highly affected by COVID-19. Finally, sources of COVID-19-related information (social media, newspaper, television, YouTube, etc.) were also assessed.

\section{Dependent variable}

Fear of COVID-19 was assessed using the Bangla Fear of COVID-19 Scale ${ }^{23}$ which was considered the dependent variable. Bangla Fear of COVID-19 Scale was adapted and validated from the English version of the scale originally developed by Ahorsu et al. ${ }^{24}$ The screening tool comprises a total of seven items (e.g., 'I am afraid of losing my life because of coronavirus-19'), and responses are recorded on a five-point Likert scale $(1=$ strongly disagree to $5=$ strongly agree). The score ranges between 7 to 35 , where a higher score indicates greater fear of COVID-19. In the present study, Cronbach's alpha was very good (0.88).

\section{Statistical analysis}

Of the 10067 respondents, data for 10052 participants were finally analysed after exclusion of 15 transgender individuals (because of a low response rate). Data were analysed using SPSS 23.0 for descriptive statistics and regression models. Independent $t$-tests and analysis of variance were performed to determine the associations between the studied variables and fear of COVID-19 (as a dependent variable). Hierarchical linear regression was used to observe the independent variables' contributions to fear of COVID-19. The normality of the distributions (e.g., skewness and kurtosis values) and multicollinearity (e.g., variance inflation factor and tolerance values) were also tested, and no issues were observed. $P<0.05$ was set as statistically significant with $95 \%$ confidence intervals. Also, gender-based and education-level-based post hoc analyses were performed for all tests. Finally, ArcGIS 10.8 was used to examine the nationwide distribution of fear of COVID-19 and its relationship with COVID-19 cases, gender and education level across districts.

\section{Ethics statement}

Ethical permission to conduct this study was granted by the Biosafety, Biosecurity and Ethical Committee of Jahangirnagar University, Bangladesh (BBEC, JU/M 2O20/COVlD-19/(9)2), and the Institute of Allergy and Clinical Immunology of Bangladesh ethics board, Bangladesh (IRBIACIB/CEC/03202005).

\section{Consent statement}

All participants provided online informed consent prior to starting the survey. They were informed about the purpose and nature of the study, and they had the right to withdraw their data if they wanted to. For participants less than 18 years old, parental consent was obtained. All participants were assured about the confidentiality of their data.

\section{Results}

\section{Characteristics of the participants}

Of the total of 10052 participants, the majority were male (56.2\%, $n$ $=5650)$, highly educated $(80.8 \%, n=8127)$, students $(58.4 \%)$ and single with respect to marital status $(70.3 \%)$, and $40.1 \%$ resided in the divisional city. About $71.3 \%$ were aged 20 to 29 years, and the mean age of the total sample was $26.95 \pm 9.632$ years (Table 1 ). About $14.7 \%$ were smokers, $2.6 \%$ reported consuming alcohol, $24.6 \%$ had a chronic disease and $90.9 \%$ used social media (Table 2). Approximately $12.9 \%$ and $2.5 \%$ of the participants reported that someone had returned to their home from the COVID-19 high-risk region Dhaka and from a foreign country, respectively. Of the COVID-19 information sources, social media was prominently reported as being used (Table 3 ).

\section{Distribution of fear of COVID-19 scores}

The overall mean score for fear of COVID-19 was $21.30 \pm 6.01$. The distribution of COVID-19 fear scores across sociodemographic variables is presented in Table 1; all of the associations were statistically significant. Female participants had higher fear of COVID-19 scores compared with their male counterparts $(22.62 \pm 5.78 v .20 .27$ $\pm 5.98 ; t=-19.913, P<0.001$ ), and highly educated participants reported higher fear of COVID-19 than less educated ones (21.47 \pm 5.87 v. $20.58 \pm 6.50 ; t=-5.856, P<0.001)$. Participants being smokers and consuming alcohol had lower scores for COVID-19 fear, whereas higher scores were found for participants with chronic diseases and those who were social media users (Table 2). Of the COVID-19-related variables, participants who used social media as a source of COVID-19 information had higher fear of COVID-19 scores $(21.52 \pm 5.86 \quad$ v. $20.27 \pm 6.55 ; \quad P<0.001)$, whereas lower scores were found for those who read newspapers for COVID-19 information (21.13 \pm 5.97 v. $21.46 \pm 6.04 ; P=$ 0.005 ) (Table 3). Gender-based and education-level-based distributions of fear of COVID-19 scores are presented in Tables 1-3.

\section{Predictive factors of fear of COVID-19}

The results of the hierarchical linear regression analysis of factors predicting fear of COVID-19 are presented in Table 4 (total sample), Supplementary Table 1 available at https://oi.org/ 10.1192/bjo.2021.984 (gender-based) and Supplementary Table 2 (education-level-based). However, sociodemographic variables were included in model 1 , whereas in model 2, behaviour- and health-related factors were compiled with sociodemographic variables. Finally, COVID-19-related variables were included in model 3 with the variables entered in model 2. All of the models were statistically significant. A 5.1\% variance of fear of COVID-19 


\begin{tabular}{|c|c|c|c|c|c|c|}
\hline \multirow[b]{2}{*}{ Study variables } & \multicolumn{2}{|c|}{ Total sample } & \multicolumn{2}{|c|}{ Gender } & \multicolumn{2}{|c|}{ Education } \\
\hline & $n, \%$ & Mean \pm s.d. & Male & Female & Lower & Higher \\
\hline \multicolumn{7}{|l|}{ Age, years } \\
\hline 10-19 & $683,6.8 \%$ & $20.46 \pm 5.95$ & $19.67 \pm 5.85$ & $21.19 \pm 5.95$ & $19.82 \pm 6.16$ & $21.58 \pm 5.39$ \\
\hline $20-29$ & 7165, 71.3\% & $21.47 \pm 5.86$ & $20.33 \pm 5.89$ & $22.91 \pm 5.50$ & $20.93 \pm 6.05$ & $21.53 \pm 5.84$ \\
\hline $30-39$ & $1218,12.1 \%$ & $21.34 \pm 6.34$ & $20.19 \pm 6.13$ & $23.38 \pm 6.18$ & $20.98 \pm 7.54$ & $21.42 \pm 6.05$ \\
\hline $40-49$ & $410,4.1 \%$ & $21.09 \pm 6.42$ & $20.50 \pm 6.26$ & $21.58 \pm 6.53$ & $21.08 \pm 6.55$ & $21.10 \pm 6.27$ \\
\hline $50-59$ & $371,3.7 \%$ & $20.37 \pm 6.33$ & $20.51 \pm 6.08$ & $20.17 \pm 6.66$ & $20.66 \pm 6.88$ & $19.95 \pm 5.43$ \\
\hline$\geq 60$ & $196,1.9 \%$ & $19.88 \pm 6.98$ & $19.58 \pm 7.01$ & $20.61 \pm 6.90$ & $19.55 \pm 7.05$ & $20.40 \pm 6.89$ \\
\hline$P$-value & - & $<0.001$ & 0.278 & $<0.001$ & 0.022 & 0.014 \\
\hline \multicolumn{7}{|l|}{ Occupation } \\
\hline Unemployed & $360,3.6 \%$ & $21.42 \pm 6.32$ & $20.89 \pm 6.33$ & $22.52 \pm 6.19$ & $19.23 \pm 7.11$ & $21.87 \pm 6.07$ \\
\hline Employed & $2586,25.7 \%$ & $21.01 \pm 6.25$ & $20.26 \pm 6.18$ & $23.25 \pm 5.92$ & $20.37 \pm 7.03$ & $21.17 \pm 6.04$ \\
\hline Retired & $91,0.9 \%$ & $20.53 \pm 6.74$ & $20.44 \pm 6.78$ & $21.08 \pm 6.71$ & $21.15 \pm 5.93$ & $20.26 \pm 7.08$ \\
\hline Housewife & $1147,11.4 \%$ & $21.83 \pm 6.41$ & $20.29 \pm 5.23$ & $22.19 \pm 6.61$ & $21.26 \pm 6.86$ & $22.24 \pm 6.02$ \\
\hline Student & $5868,58.4 \%$ & $21.33 \pm 5.77$ & $20.22 \pm 5.85$ & $22.64 \pm 5.39$ & $20.39 \pm 5.88$ & $21.48 \pm 5.74$ \\
\hline$P$-value & - & 0.003 & 0.563 & 0.008 & 0.051 & $<0.001$ \\
\hline \multicolumn{7}{|l|}{ Residence } \\
\hline Village & 2330, 23.2\% & $20.89 \pm 6.38$ & $20.41 \pm 6.22$ & $21.97 \pm 6.59$ & $20.01 \pm 7.17$ & $21.27 \pm 5.96$ \\
\hline Upazila town & $1358,13.5 \%$ & $21.57 \pm 5.85$ & $20.69 \pm 5.91$ & $22.94 \pm 5.49$ & $21.06 \pm 6.00$ & $21.69 \pm 5.81$ \\
\hline District town & $2330,23.2 \%$ & $21.56 \pm 5.91$ & $20.32 \pm 5.95$ & $22.81 \pm 5.59$ & $21.05 \pm 6.12$ & $21.68 \pm 5.85$ \\
\hline Divisional town & $4034,40.1 \%$ & $21.29 \pm 5.88$ & $19.96 \pm 5.82$ & $22.67 \pm 5.63$ & $20.71 \pm 6.02$ & $21.38 \pm 5.86$ \\
\hline$P$-value & - & $<0.001$ & 0.014 & 0.007 & 0.023 & 0.084 \\
\hline \multicolumn{7}{|l|}{ Administrative division } \\
\hline Barishal & $207,2.1 \%$ & $21.49 \pm 6.91$ & $20.05 \pm 7.32$ & $23.65 \pm 5.64$ & $23.00 \pm 8.15$ & $21.17 \pm 6.61$ \\
\hline Chittagong & $2406,23.9 \%$ & $21.38 \pm 5.94$ & $20.40 \pm 5.93$ & $22.74 \pm 5.69$ & $21.24 \pm 6.39$ & $21.42 \pm 5.83$ \\
\hline Dhaka & $4286,42.6 \%$ & $21.53 \pm 5.81$ & $20.28 \pm 5.76$ & $22.92 \pm 5.54$ & $20.94 \pm 5.82$ & $21.64 \pm 5.80$ \\
\hline Khulna & $1044,10.4 \%$ & $20.62 \pm 6.52$ & $19.85 \pm 6.50$ & $21.62 \pm 6.41$ & $18.67 \pm 7.35$ & $21.43 \pm 5.96$ \\
\hline Mymensingh & $256,2.5 \%$ & $21.37 \pm 6.06$ & $20.10 \pm 6.15$ & $23.33 \pm 5.40$ & $21.60 \pm 5.28$ & $21.33 \pm 6.21$ \\
\hline Rajshahi & $944,9.4 \%$ & $21.11 \pm 6.08$ & $20.52 \pm 5.97$ & $22.04 \pm 6.15$ & $20.94 \pm 6.71$ & $21.16 \pm 5.90$ \\
\hline Rangpur & $558,5.6 \%$ & $20.42 \pm 6.09$ & $19.99 \pm 5.91$ & $21.31 \pm 6.37$ & $18.47 \pm 6.49$ & $21.06 \pm 5.82$ \\
\hline Sylhet & $333,3.3 \%$ & $21.55 \pm 5.99$ & $20.32 \pm 6.19$ & $22.92 \pm 5.46$ & $22.24 \pm 5.83$ & $21.40 \pm 6.02$ \\
\hline$P$-value & - & $<0.001$ & 0.576 & $<0.001$ & $<0.001$ & 0.345 \\
\hline \multicolumn{7}{|l|}{ Marital status } \\
\hline Single & 7071, 70.3\% & $21.20 \pm 5.79$ & $20.23 \pm 5.83$ & $22.62 \pm 5.44$ & $20.43 \pm 6.04$ & $21.32 \pm 5.74$ \\
\hline Married & $2838,28.2 \%$ & $21.56 \pm 6.46$ & $20.40 \pm 6.37$ & $22.74 \pm 6.35$ & $20.82 \pm 6.92$ & $21.88 \pm 6.24$ \\
\hline Divorced/widowed/other & $143,1.4 \%$ & $20.90 \pm 6.80$ & $19.78 \pm 7.35$ & $21.35 \pm 6.56$ & $19.93 \pm 7.27$ & $22.37 \pm 5.79$ \\
\hline$P$-value & - & 0.016 & 0.571 & 0.064 & 0.284 & 0.001 \\
\hline
\end{tabular}

scores was predicted in the total sample; this increased to $5.7 \%$ and $6.2 \%$ in model 2 and model 3, respectively. The gender-based and education-level-based explanatory power of fear of COVID-19 can be found in Supplementary Tables 1 and 2, respectively. The final gender-based hierarchical regression model explained 2\% and $1.9 \%$ of the variance in predicted fear of COVID-19 for males and females, respectively (Supplementary Table 1), whereas $4.7 \%$ and $6.7 \%$ of the variance were explained in the final educationlevel model for lower and higher education levels, respectively (Supplementary Table 2).

\section{District-wise distribution of fear of COVID-19}

District-wise spatial distribution of fear of COVID-19 is presented in Fig. 1(a) and was statistically significant $(F=5.897, P<0.001)$. Higher levels of fear of COVID-19 were found in Magura, Panchagarh, Tangail, Sunamganj, Munshiganj, Bogra, Barguna, Narail and Naraynganj; by contrast, Kushtia, Pirojpur, Chapainawabganj, Jhalokathi, Naogaon and Rangpur districts had lower fear of COVID-19.

\section{District-wise relationship of fear of COVID-19 with COVID-19 cases}

Figure 1(b) represents the relationship of fear of COVID-19 with numbers of COVID-19 cases in the respective districts. However, fear of COVID-19 and COVID-19 cases were heterogeneously distributed across the districts; that is, no consistent association of higher COVID-19 cases with higher fear of COVID-19 was found.

\section{District-wise distribution of gender-based fear of COVID-19}

The gender-based distribution of fear of COVID-19 across districts is presented in Fig. 1(c); the associations of both genders with fear of COVID-19 were found to be statistically significant $(F=2.932, P<$ 0.001 , and $F=4.415, P<0.001$ for males and females, respectively). Male participants from the districts of Magura, Munshiganj, Sunamganj, Bandarban, Feni, Tangail, Panchagarh and Bogra had higher fear of COVID-19 scores, as did female participants from Patuakhali, Joypurhat, Manikganj, Lakshmipur, Panchagarh, Barishal, Tangail and Barguna. However, both genders from the districts of Panchagarh, Bogra, Tangail and Sunamganj reported higher fear of COVID-19.

\section{District-wise distribution of education-level-based fear of COVID-19}

The education-level-based distribution of fear of COVID-19 across districts is presented in Fig. 1(d); the associations of both education levels with fear of COVID-19 were found to be statistically significant $(F=6.226, P<0.001$, and $F=1.773, P<0.001$ for lower education and higher education, respectively). Participants with lower education from the districts of Lalmonirhat, Barishal, Bandarban, Tangail, Sirajganj, Magura, Bogra, Natore and Gaibandha had 


\begin{tabular}{|c|c|c|c|c|c|c|}
\hline \multirow[b]{2}{*}{ Study variables } & \multicolumn{2}{|c|}{ Total sample } & \multicolumn{2}{|c|}{ Gender } & \multicolumn{2}{|c|}{ Education } \\
\hline & $n, \%$ & Mean \pm s.d. & Male & Female & Lower & Higher \\
\hline \multicolumn{7}{|l|}{ Current smoker } \\
\hline Yes & $1478,14.7 \%$ & $20.29 \pm 6.18$ & $20.20 \pm 6.17$ & $22.98 \pm 6.32$ & $19.94 \pm 6.63$ & $20.39 \pm 6.07$ \\
\hline No & $8574,85.3 \%$ & $21.47 \pm 5.95$ & $20.29 \pm 5.92$ & $22.62 \pm 5.77$ & $20.70 \pm 6.47$ & $21.65 \pm 5.82$ \\
\hline$P$-value & - & $<0.001$ & 0.612 & 0.659 & 0.059 & $<0.001$ \\
\hline \multicolumn{7}{|l|}{ Alcohol use } \\
\hline Yes & $264,2.6 \%$ & $19.85 \pm 6.35$ & $19.62 \pm 6.32$ & $22.29 \pm 6.27$ & $18.78 \pm 6.84$ & $20.23 \pm 6.15$ \\
\hline No & $9788,97.4 \%$ & $21.33 \pm 5.99$ & $20.29 \pm 5.96$ & $22.63 \pm 5.77$ & $20.64 \pm 6.48$ & $21.50 \pm 5.86$ \\
\hline$P$-value & - & $<0.001$ & 0.085 & 0.776 & 0.021 & 0.003 \\
\hline \multicolumn{7}{|l|}{ Perceived health status } \\
\hline very good & $6899,68.6 \%$ & $20.96 \pm 6.02$ & $19.94 \pm 5.94$ & $22.37 \pm 5.86$ & $20.05 \pm 6.62$ & $21.14 \pm 5.88$ \\
\hline Acceptable & $2807,27.9 \%$ & $22.06 \pm 5.83$ & $21.04 \pm 5.92$ & $23.19 \pm 5.53$ & $21.29 \pm 6.20$ & $22.30 \pm 5.69$ \\
\hline Poor & $312,3.1 \%$ & $21.85 \pm 6.34$ & $21.16 \pm 6.61$ & $22.56 \pm 5.99$ & $21.67 \pm 6.28$ & $21.97 \pm 6.39$ \\
\hline very poor & $34,0.3 \%$ & $21.50 \pm 7.25$ & $20.94 \pm 8.08$ & $22.12 \pm 6.39$ & $21.09 \pm 8.32$ & $21.69 \pm 6.87$ \\
\hline$P$-value & - & $<0.001$ & $<0.001$ & $<0.001$ & $<0.001$ & $<0.001$ \\
\hline \multicolumn{7}{|l|}{ Comorbidity status } \\
\hline No & $7581,75.4 \%$ & $21.15 \pm 5.96$ & $20.04 \pm 5.94$ & $22.61 \pm 5.68$ & $20.17 \pm 6.57$ & $21.34 \pm 5.82$ \\
\hline Yes & $2471,24.6 \%$ & $21.75 \pm 6.10$ & $20.99 \pm 6.06$ & $22.67 \pm 6.04$ & $21.31 \pm 6.30$ & $21.92 \pm 6.03$ \\
\hline$P$-value & - & $<0.001$ & $<0.001$ & $<0.001$ & $<0.001$ & $<0.001$ \\
\hline \multicolumn{7}{|l|}{ Social media use } \\
\hline Yes & $9139,90.9 \%$ & $21.40 \pm 5.87$ & $20.29 \pm 5.88$ & $22.88 \pm 5.52$ & $20.90 \pm 6.00$ & $21.48 \pm 5.85$ \\
\hline No & $913,9.1 \%$ & $20.26 \pm 7.13$ & $19.91 \pm 7.02$ & $20.58 \pm 7.22$ & $20.01 \pm 7.26$ & $21.06 \pm 6.64$ \\
\hline$P$-value & - & $<0.001$ & 0.196 & $<0.001$ & 0.004 & 0.302 \\
\hline \multicolumn{7}{|l|}{ Social media use frequency } \\
\hline More than 4 days per week & $292,2.9 \%$ & $21.51 \pm 6.18$ & $20.50 \pm 6.27$ & $23.08 \pm 5.72$ & $22.42 \pm 6.39$ & $21.25 \pm 6.11$ \\
\hline 2-3 days per week & $317,3.2 \%$ & $20.99 \pm 5.56$ & $20.05 \pm 5.95$ & $22.18 \pm 4.79$ & $22.17 \pm 5.73$ & $20.41 \pm 5.40$ \\
\hline Every day & $4077,40.6 \%$ & $21.42 \pm 5.83$ & $20.40 \pm 5.84$ & $22.95 \pm 5.47$ & $20.63 \pm 5.94$ & $21.55 \pm 5.80$ \\
\hline Several times per day & $4443,44.2 \%$ & $21.45 \pm 5.92$ & $20.28 \pm 5.90$ & $22.86 \pm 5.63$ & $20.82 \pm 6.07$ & $21.54 \pm 5.89$ \\
\hline$P$-value & - & 0.583 & 0.764 & 0.439 & 0.02 & 0.04 \\
\hline
\end{tabular}

\begin{tabular}{|c|c|c|c|c|c|c|}
\hline \multirow[b]{2}{*}{ Study variables } & \multicolumn{2}{|c|}{ Total sample } & \multicolumn{2}{|c|}{ Gender } & \multicolumn{2}{|c|}{ Education } \\
\hline & $n, \%$ & Mean \pm s.d. & Male & Female & Lower & Higher \\
\hline \multicolumn{7}{|c|}{ Someone returns home from Dhaka } \\
\hline Yes & $1292,12.9 \%$ & $21.14 \pm 6.01$ & $20.15 \pm 5.99$ & $22.62 \pm 5.72$ & $20.82 \pm 6.66$ & $21.22 \pm 5.83$ \\
\hline No & $7660,76.2 \%$ & $21.37 \pm 5.99$ & $20.36 \pm 5.98$ & $22.66 \pm 5.78$ & $20.55 \pm 6.48$ & $21.57 \pm 5.86$ \\
\hline$P$-value & - & 0.208 & 0.380 & 0.870 & 0.540 & 0.074 \\
\hline \multicolumn{7}{|c|}{ Someone returns home from a COVID-19-affected country } \\
\hline Yes & $254,2.5 \%$ & $21.17 \pm 6.06$ & $19.94 \pm 5.76$ & $22.59 \pm 6.11$ & $20.05 \pm 6.38$ & $21.49 \pm 5.94$ \\
\hline No & $9798,97.5 \%$ & $21.30 \pm 6.01$ & $20.27 \pm 5.98$ & $22.63 \pm 5.77$ & $20.59 \pm 6.50$ & $21.47 \pm 5.87$ \\
\hline$P$-value & - & 0.733 & 0.520 & 0.951 & 0.538 & 0.962 \\
\hline \multicolumn{7}{|c|}{ Source of COVID-19 information: social media } \\
\hline No & $1784,17.7 \%$ & $20.27 \pm 6.55$ & $19.36 \pm 6.49$ & $21.27 \pm 6.47$ & $20.19 \pm 7.06$ & $20.33 \pm 6.05$ \\
\hline Yes & $8268,82.3 \%$ & $21.52 \pm 5.86$ & $20.45 \pm 5.86$ & $22.95 \pm 5.55$ & $20.89 \pm 5.99$ & $21.62 \pm 5.83$ \\
\hline$P$-value & - & $<0.001$ & $<0.001$ & $<0.001$ & 0.02 & $<0.001$ \\
\hline \multicolumn{7}{|c|}{ Source of COVID-19 information: YouTube } \\
\hline No & $2755,27.4 \%$ & $21.32 \pm 6.02$ & $20.22 \pm 5.97$ & $22.45 \pm 5.86$ & $20.65 \pm 6.59$ & $21.52 \pm 5.82$ \\
\hline Yes & $7297,72.6 \%$ & $21.27 \pm 5.99$ & $20.32 \pm 5.99$ & $22.94 \pm 5.61$ & $20.43 \pm 6.27$ & $21.41 \pm 5.93$ \\
\hline$P$-value & - & 0.721 & 0.548 & 0.007 & 0.493 & 0.411 \\
\hline \multicolumn{7}{|c|}{ Source of COVID-19 information: newspaper } \\
\hline No & $5124,51.0 \%$ & $21.46 \pm 6.04$ & $20.31 \pm 6.04$ & $22.65 \pm 5.81$ & $20.64 \pm 6.64$ & $21.72 \pm 5.82$ \\
\hline Yes & $4928,49.0 \%$ & $21.13 \pm 5.97$ & $20.23 \pm 5.93$ & $22.58 \pm 5.73$ & $20.48 \pm 6.26$ & $21.24 \pm 5.91$ \\
\hline$P$-value & - & 0.005 & 0.574 & 0.688 & 0.593 & $<0.001$ \\
\hline \multicolumn{7}{|c|}{ Source of COVID-19 information: television } \\
\hline No & $2755,27.4 \%$ & $21.24 \pm 6.01$ & $20.16 \pm 5.99$ & $22.65 \pm 5.72$ & $20.37 \pm 6.43$ & $21.40 \pm 5.91$ \\
\hline Yes & $7297,72.6 \%$ & $21.32 \pm 6.01$ & $20.31 \pm 5.97$ & $22.62 \pm 5.79$ & $20.64 \pm 6.52$ & $21.50 \pm 5.86$ \\
\hline$P$-value & - & 0.531 & 0.396 & 0.863 & 0.451 & 0.514 \\
\hline \multicolumn{7}{|c|}{ Source of COVID-19 information: health website } \\
\hline No & $5559,55.3 \%$ & $21.35 \pm 6.06$ & $20.32 \pm 6.04$ & $22.55 \pm 5.86$ & $20.58 \pm 6.69$ & $21.63 \pm 5.79$ \\
\hline Yes & $4493,44.7 \%$ & $21.24 \pm 5.94$ & $20.20 \pm 5.91$ & $22.73 \pm 5.65$ & $20.56 \pm 5.80$ & $21.31 \pm 5.95$ \\
\hline$P$-value & - & 0.330 & 0.440 & 0.292 & 0.948 & 0.014 \\
\hline \multicolumn{7}{|c|}{ Source of COVID-19 information: other sources } \\
\hline No & $8106,80.6 \%$ & $21.46 \pm 5.92$ & $20.34 \pm 5.92$ & $22.79 \pm 5.65$ & $20.78 \pm 6.42$ & $21.61 \pm 5.79$ \\
\hline Yes & $1946,19.4 \%$ & $20.63 \pm 6.29$ & $20.03 \pm 6.18$ & $21.75 \pm 6.34$ & $19.93 \pm 6.69$ & $20.85 \pm 6.15$ \\
\hline$P$-value & - & $<0.001$ & 0.106 & $<0.001$ & 0.015 & $<0.001$ \\
\hline
\end{tabular}




\begin{tabular}{|c|c|c|c|c|c|c|}
\hline Models & & B & s.e. & $\beta$ & $t$ & $P$ \\
\hline \multirow{8}{*}{$\begin{array}{l}\text { Model } 1 \\
\qquad R^{2}=0.051, \text { Adjusted } R^{2}=0.050, P<0.001\end{array}$} & (Constant) & 18.071 & 0.604 & & 29.903 & $<0.001$ \\
\hline & Age & -0.026 & 0.013 & -0.030 & -2.022 & 0.043 \\
\hline & Gender $^{a}$ & 2.594 & 0.136 & 0.219 & 19.138 & $<0.001$ \\
\hline & Education $^{\mathrm{b}}$ & 0.526 & 0.185 & 0.031 & 2.843 & 0.004 \\
\hline & Occupation ${ }^{c}$ & -0.099 & 0.056 & -0.023 & -1.758 & 0.079 \\
\hline & Residence $^{d}$ & -0.215 & 0.056 & -0.043 & -3.838 & $<0.001$ \\
\hline & Administrative division & -0.081 & 0.037 & -0.024 & -2.196 & 0.028 \\
\hline & Marital status ${ }^{f}$ & 0.542 & 0.186 & 0.041 & 2.907 & 0.004 \\
\hline \multirow{14}{*}{$\begin{array}{l}\text { Model } 2 \\
\qquad R^{2}=0.057, \text { Adjusted } R^{2}=0.056, P<0.001\end{array}$} & (constant) & 15.645 & 1.261 & & 12.411 & $<0.001$ \\
\hline & Age & -0.039 & 0.013 & -0.046 & -3.001 & 0.003 \\
\hline & Gender $^{a}$ & 2.499 & 0.142 & 0.211 & 17.567 & $<0.001$ \\
\hline & Education $^{\mathrm{b}}$ & 0.659 & 0.186 & 0.039 & 3.540 & $<0.001$ \\
\hline & Occupation ${ }^{c}$ & -0.118 & 0.056 & -0.028 & -2.101 & 0.036 \\
\hline & Residence $^{d}$ & -0.206 & 0.056 & -0.042 & -3.663 & $<0.001$ \\
\hline & Administrative division ${ }^{e}$ & -0.080 & 0.037 & -0.024 & -2.172 & 0.030 \\
\hline & Marital status ${ }^{f}$ & 0.529 & 0.186 & 0.040 & 2.843 & 0.004 \\
\hline & Smoking status ${ }^{g}$ & 0.188 & 0.205 & 0.011 & 0.916 & 0.359 \\
\hline & Alcohol consumptiong & 0.379 & 0.431 & 0.010 & 0.879 & 0.380 \\
\hline & Perceived health status ${ }^{h}$ & 0.393 & 0.067 & 0.065 & 5.836 & $<0.001$ \\
\hline & Comorbidity status & 0.458 & 0.162 & 0.033 & 2.832 & 0.005 \\
\hline & Social media userg & 1.153 & 0.654 & 0.019 & 1.764 & 0.078 \\
\hline & Social media use frequency ${ }^{j}$ & -0.085 & 0.090 & -0.010 & -0.939 & 0.348 \\
\hline \multirow{22}{*}{$\begin{array}{l}\text { Model } 3 \\
\qquad R^{2}=0.062, \text { Adjusted } R^{2}=0.059, P<0.001\end{array}$} & (constant) & 14.428 & 1.529 & & 9.438 & $<0.001$ \\
\hline & Age & -0.037 & 0.013 & -0.043 & -2.820 & 0.005 \\
\hline & Gender $^{a}$ & 2.452 & 0.144 & 0.207 & 17.063 & $<0.001$ \\
\hline & Education $^{\mathrm{b}}$ & 0.655 & 0.187 & 0.039 & 3.493 & $<0.001$ \\
\hline & Occupation $^{c}$ & -0.120 & 0.056 & -0.028 & -2.128 & 0.033 \\
\hline & Residence $^{d}$ & -0.201 & 0.057 & -0.041 & -3.516 & $<0.001$ \\
\hline & Administrative division ${ }^{e}$ & -0.072 & 0.037 & -0.021 & -1.957 & 0.050 \\
\hline & Marital status ${ }^{f}$ & 0.493 & 0.186 & 0.037 & 2.645 & 0.008 \\
\hline & Smoking status ${ }^{g}$ & 0.174 & 0.205 & 0.010 & 0.848 & 0.396 \\
\hline & Alcohol consumptiong & 0.354 & 0.431 & 0.009 & 0.820 & 0.412 \\
\hline & Perceived health status ${ }^{\text {h }}$ & 0.386 & 0.067 & 0.064 & 5.729 & $<0.001$ \\
\hline & Comorbidity status ${ }^{i}$ & 0.485 & 0.162 & 0.035 & 2.996 & 0.003 \\
\hline & Social media userg & 1.387 & 0.655 & 0.023 & 2.119 & 0.034 \\
\hline & Social media use frequency ${ }^{j}$ & -0.126 & 0.091 & -0.015 & -1.382 & 0.167 \\
\hline & SRHF Dhakag & 0.324 & 0.185 & 0.019 & 1.754 & 0.080 \\
\hline & SRHF COVID-19-infected country ${ }^{g}$ & -0.124 & 0.409 & -0.003 & -0.302 & 0.763 \\
\hline & sCl: social media' & 1.056 & 0.213 & 0.056 & 4.946 & $<0.001$ \\
\hline & SCl: YouTubei & 0.095 & 0.140 & 0.008 & 0.677 & 0.498 \\
\hline & SCl: Newspaper ${ }^{i}$ & -0.271 & 0.145 & -0.023 & -1.868 & 0.062 \\
\hline & SCl: television' & 0.189 & 0.153 & 0.015 & 1.232 & 0.218 \\
\hline & SCl: health website ${ }^{i}$ & -0.087 & 0.137 & -0.007 & -0.634 & 0.526 \\
\hline & SCl: other sources ${ }^{i}$ & -0.400 & 0.173 & -0.026 & -2.313 & 0.021 \\
\hline \multicolumn{7}{|c|}{ 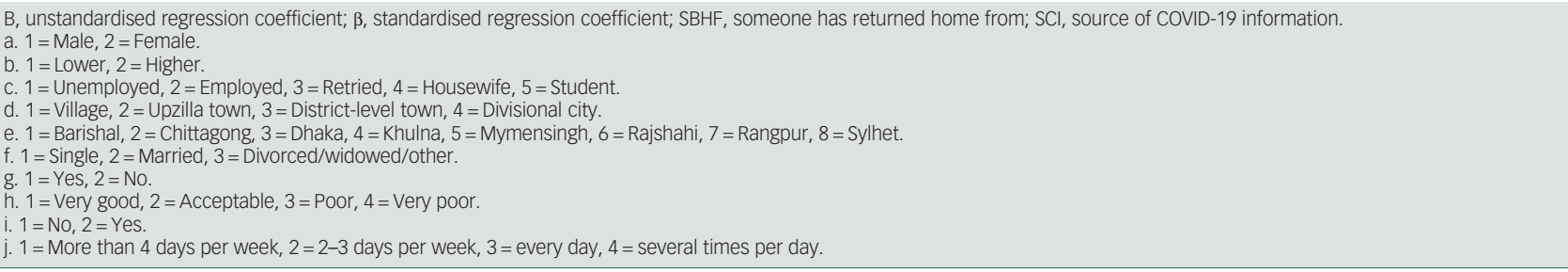 } \\
\hline
\end{tabular}

higher fear of COVID-19 scores, compared with those from Sunamganj, Panchagarh, Magura, Munshiganj, Barguna Naraynganj, Tangail, Kurigram and Shariatpur for more highly educated participants. However, participants of both education levels from the districts of Tangail, Magura, Bogra and Gaibandha had higher fear of COVID-19 scores.

\section{Discussion}

The COVID-19 pandemic is a public health threat that can increase peoples' psychological risk and vulnerability by intensifying mental health stressors including fear, panic and uncertainty. Furthermore, the unexpected fear of COVID-19 can be life-threatening by leading to a drastic decision such as suicide. ${ }^{5-8}$ Therefore, identifying the factors associated with fear of COVID-19 can help with the adoption and implementation of a better mental health strategy. Herein, factors related to fear of COVID-19 were identified from a nationwide sample collected by an online survey during the first wave of the pandemic in Bangladesh. The sample was limited owing to the use of an online survey with responses overwhelmingly from educated individuals; therefore, a post hoc analysis considering participants' education levels was conducted. This was the first attempt to present a GIS-based distribution of fear of COVID-19 across the administrative districts of the country.

The overall mean fear of COVID-19 score was $21.30 \pm 6.01$ (out of a possible of 35) in the present sample. Use of the same instrument to assess levels of fear of COVID-19 has been reported in 
(a)

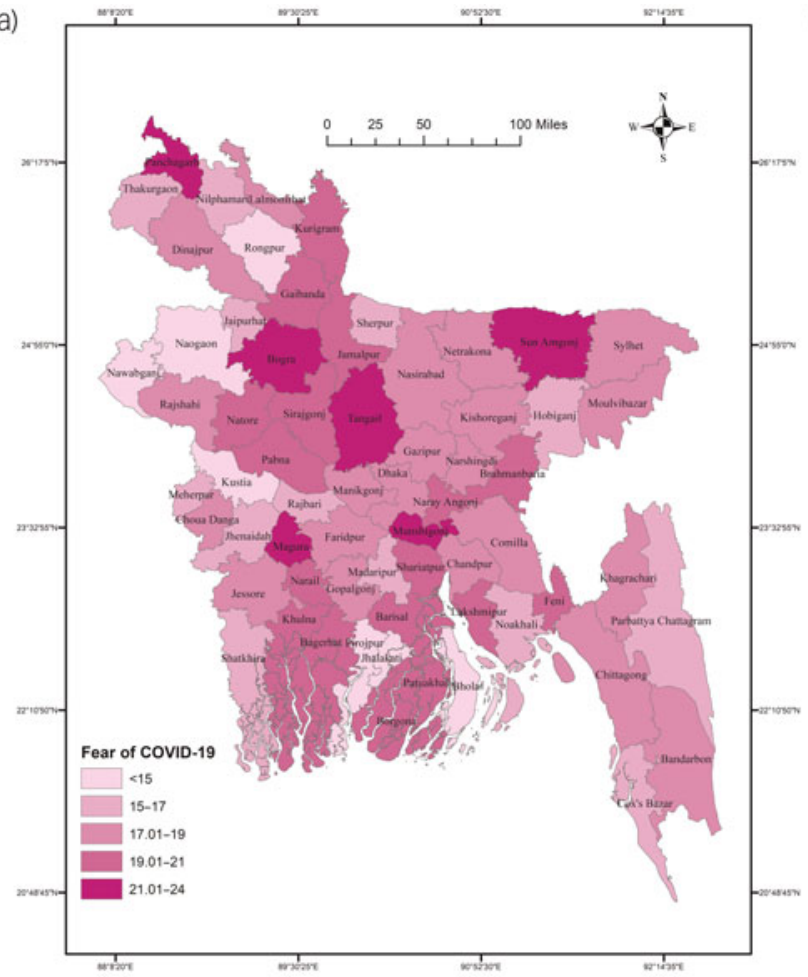

(c)

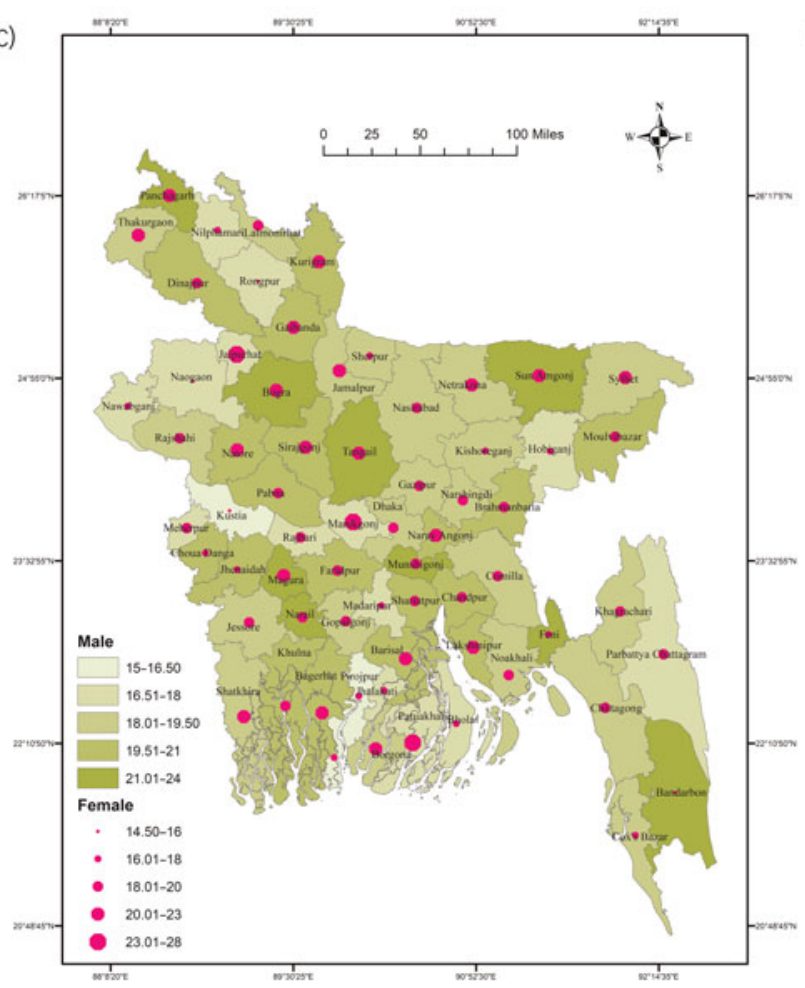

(b)

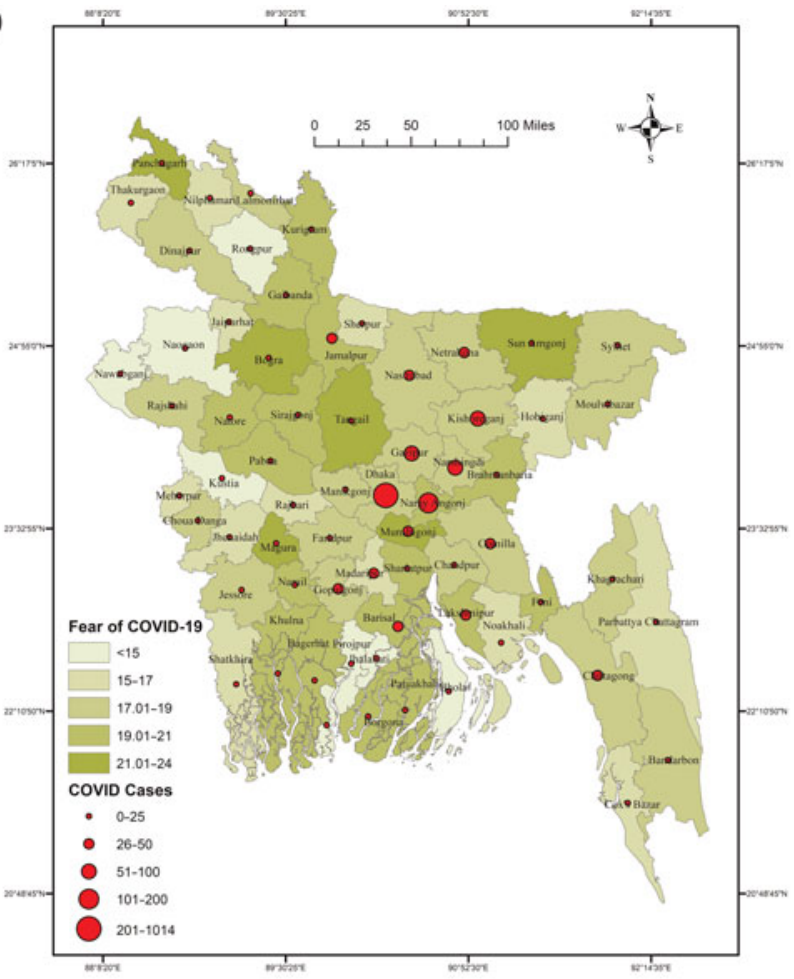

(d)

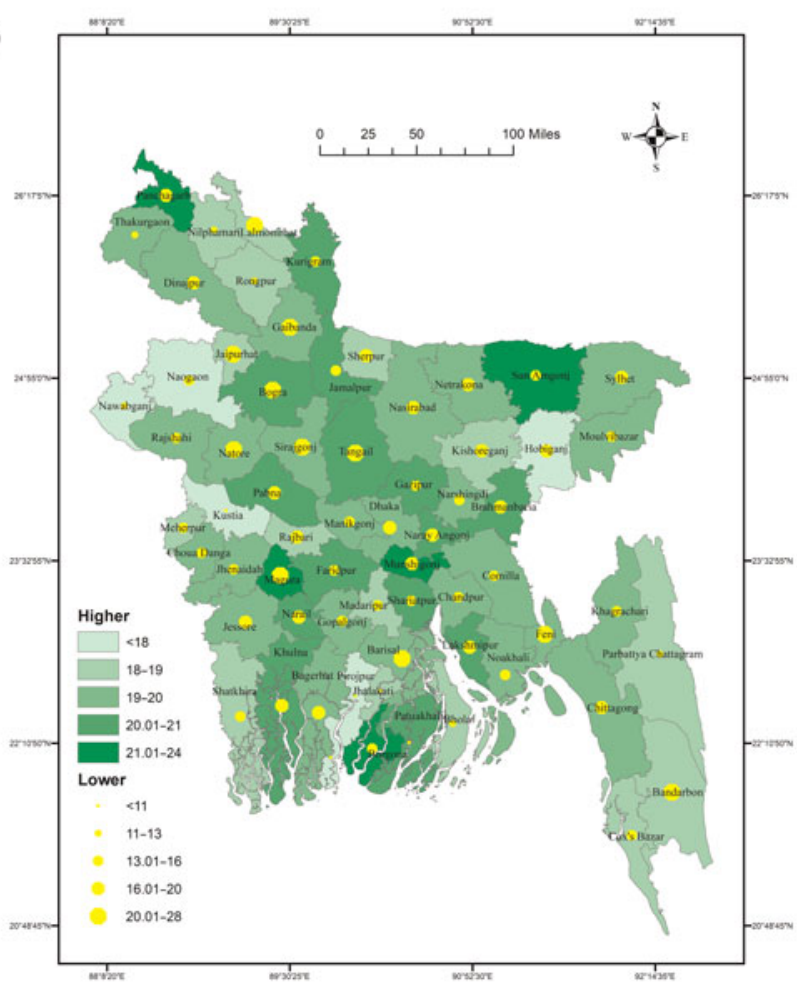

Fig. 1 (a) Distribution of fear of COVID-19 across districts in Bangladesh. (b) Distribution of fear of COVID-19 and COVID-19 cases across districts (c) Gender-based distribution of fear of COVID-19 across districts. (d) Education-level-based distribution of fear of COVID-19 across districts.

other Bangladeshi studies. For example, a mean score of $18.53 \pm$ 5.013 was reported for a survey conducted from April to May 2020 in a sample of 2157 individuals, ${ }^{12}$ and $19.4 \pm 6.1$ in another study comprising a total of 1032 elderly individuals conducted in October $2020{ }^{13}$ Thus, the present sample showed higher fear of COVID-19 scores, although comparisons were limited because of the study implementation time and lack of representativeness of samples across these Bangladeshi studies.

The study found female gender to be a risk factor for reporting higher fear of COVID-19; similarly, gender was identified as one of the significant predictors of fear of COVID-19 in other Bangladeshi studies. ${ }^{4,12,13}$ Reckless and irresponsible attitudes towards COVID- 
19 are more frequently observed among male participants, indicating that females are more conscious of preventive COVID-19 behaviours, leading to higher fear of COVID-19. ${ }^{16}$ Similarly, the level of consciousness increases with higher levels of educational attainment. That is, more educated individuals are at risk of higher fear of COVID-19 owing to the avoidance behaviours they adopt to protect themselves from potential virus infection, ${ }^{16-18}$ which is also reported in the study.

The studied variables predicted the explanatory power of the fear of COVID-19 in this study. Initially, sociodemographic variables (i.e., age, gender, education, occupation, residence, administrative division and marital status) predicted a $5.1 \%$ variance in fear of COVID-19. When behaviour- and health-related factors (i. e., smoking status, alcohol consumption, perceived health status, comorbidity status, social media use and frequency of social media use) were included, together with sociodemographic variables, the variance in fear of COVID-19 increased to 5.7\%. Finally, after adjusting for COVID-19-related variables, an additional $0.5 \%$ of explanatory power was obtained. As this was an exploratory study, comparisons of the present models with those of prior studies were limited. However, it is anticipated that these models will help to facilitate further investigations.

The GIS-based distribution-related all of the associations with fear of COVID-19 were statistically significant. Higher fear of COVID-19 was found in the districts of Magura, Panchagarh, Tangail, Sunamganj, Munshiganj, Bogra, Barguna, Narail and Naraynganj. However, with respect to the relationship between COVID-19 fear and numbers of COVID-19 cases across districts, a heterogeneous distribution was found; that is, there was no consistent relationship between higher or lower COVID-19 case numbers with higher or lower fear of COVID-19. The districts of Panchagarh, Bogra, Tangail and Sunamganj reported higher fear of COVID-19 for both genders, although there were distinct districts reporting higher COVID-19 fear for each gender. Similarly, the districts of Tangail, Magura, Bogra and Gaibandha had higher fear of COVID-19 scores for participants of both education levels.

Several methodological issues of the present study should be noted as limitations. First, it was a cross-sectional study; this may hinder the ability to infer causal associations from the current findings. Online surveys can be limited by selection bias (e.g., the overwhelming response from educated persons). Moreover, important behavioural predictors influencing the level of fear of COVID-19 (perceived behavioural control, action self-efficacy, etc.) were not considered in this study. ${ }^{25}$ However, despite these limitations, this study provides baseline information across districts on an important but unexplored issue, fear of COVID-19, in the context of Bangladesh.

Mohammed A. Mamun (D), Director, CHINTA Research Bangladesh, Savar, Dhaka, Bangladesh, and Department of Public Health and Informatics, Jahangirnagar University, Savar, Dhaka, Bangladesh

Correspondence: Mohammed A. Mamun. Email: mamunphi46@gmail.com

First received 15 May 2021, final revision 2 Jul 2021, accepted 27 Jul 2021

\section{Supplementary material}

Supplementary material is available online at https://doi.org/10.1192/bjo.2021.984.

\section{Data availability}

The data used in this study can be accessed at: https://doi.org/10.1016/j.dib.2020.106621.

\section{Acknowledgements}

The author thanks all the participants and research assistants, without whom this self-funded study could not have been implemented. It should also be noted that the author's affiliation, the study could not have been implemented. It should also be noted that the author's affiliation, the
CHINTA Research Bangladesh, was formerly known as the Undergraduate Research Organisation.

\section{Funding}

None.

Declaration of interest

None.

\section{References}

1 Mertens G, Gerritsen L, Duijndam S, Salemink E, Engelhard IM. Fear of the coronavirus (COVID-19): predictors in an online study conducted in March 2020. J Anxiety Disord 2020; 74: 102258.

2 Mahmud MS, Talukder MU, Rahman SM. Does "Fear of COVID-19" trigger future career anxiety? An empirical investigation considering depression from COVID19 as a mediator. Int J Soc Psychiatry 2020; 67(1): 35-45.

3 Mamun MA, Griffiths MD. First COVID-19 suicide case in Bangladesh due to fear of COVID-19 and xenophobia: possible suicide prevention strategies. Asian J Psychiatr 2020; 51: e102073.

4 Sakib N, Akter T, Zohra F, Bhuiyan AKMI, Mamun MAMA, Griffiths MD. Fear of COVID-19 and depression: a comparative study among the general population and healthcare professionals during COVID-19 pandemic crisis in Bangladesh. Int J Ment Health Addict [Epub ahead of print] 19 Feb 2021. Available from: https://doi.org/10.1007/s11469-020-00477-9.

5 Dsouza DD, Quadros S, Hyderabadwala ZJ, Mamun MA. Aggregated COVID-19 suicide incidences in India: fear of COVID-19 infection is the prominent causative factor. Psychiatry Res 2020; 290: e113145.

6 Griffiths MD, Mamun MA. COVID-19 suicidal behavior among couples and suicide pacts: case study evidence from press reports. Psychiatry Res 2020; 289: 113105.

7 Cheung YT, Chau PH, Yip PSF. A revisit on older adults suicides and Severe Acute Respiratory Syndrome (SARS) epidemic in Hong Kong. Int J Geriatr Psychiatry 2008; 23: 1231-8

8 Yip PSF, Cheung YT, Chau PH, Law YW. The impact of epidemic outbreak: the case of severe acute respiratory syndrome (SARS) and suicide among older adults in Hong Kong. Cris J Cris Interv Suicide Prev 2010; 31: 86.

9 Mamun MA. Suicide and suicidal behaviors in the context of COVID-19 pandemic in Bangladesh: a systematic review. Psychol Res Behav Manag 2021; 14: 695-704.

10 al Mamun F, Hosen I, Misti JM, Kaggwa MM, Mamun MA. Mental disorders of Bangladeshi students during the COVID-19 pandemic: a systematic review. Psychol Res Behav Manag 2021; 14: 645-54.

11 Hossain MM, Rahman M, Trisha NF, Tasnim S, Nuzhath T, Hasan NT, et al. Prevalence of anxiety and depression in South Asia during COVID-19: a systematic review and meta-analysis. Heliyon 2021; 7(4): e06677.

12 Hossain MA, Hossain KMA, Walton LM, Uddin Z, Haque MO, Kabir MF, et al. Knowledge, attitudes, and fear of COVID-19 during the rapid rise period in Bangladesh. PLOS One 2020; 15(9): e0239646.

13 Mistry SK, Ali ARMM, Akther F, Yadav UN, Harris MF. Exploring fear of COVID-19 and its correlates among older adults in Bangladesh. Global Health 2021; 17: 47.

14 Harper CA, Satchell LP, Fido D, Latzman RD. Functional fear predicts public health compliance in the COVID-19 pandemic. Int J Ment Health Addict [Epub ahead of print] 27 Apr 2020. Available from: https://doi.org/10.1007/s11469020-00281-5.

15 Pakpour AH, Griffiths MD. The fear of COVID-19 and its role in preventive behaviors. J Concurr Disord 2020; 2: 58-63.

16 Islam SMDU, Safiq MB, Bodrud-Doza M, Mamun MA. Perception and attitudes towards PPE-related waste disposal amid COVID-19 in Bangladesh: an exploratory study. Front Public Health 2020; 8: 699.

17 Zhong B-L, Luo W, Li H-M, Zhang Q-Q, Liu X-G, Li W-T, et al. Knowledge, attitudes, and practices towards COVID-19 among Chinese residents during the rapid rise period of the COVID-19 outbreak: a quick online cross-sectional survey. Int J Biol Sci 2020; 16: 1745-52.

18 Al-Hanawi MK, Angawi K, Alshareef N, Qattan AMN, Helmy HZ, Abudawood Y, et al. Knowledge, attitude and practice toward COVID-19 among the public in the Kingdom of Saudi Arabia: a cross-sectional study. Front Public Health 2020; 8: 217. 
19 Pakpour AH, Al Mamun F, Hosen I, Griffiths MD, Mamun MA. A populationbased nationwide dataset concerning the COVID-19 pandemic and serious psychological consequences in Bangladesh. Data Brief 2020; 33: 106621

20 Mamun MA, Sakib N, Gozal D, Bhuiyan AKMI, Hossain S, Bodrud-Doza M, et al. The COVID-19 pandemic and serious psychological consequences in Bangladesh: a population-based nationwide study. J Affect Disord 2021; 279: 462-72.

21 al Mamun F, Gozal D, Hosen I, Misti JM, Mamun MA. Predictive factors of insomnia during the COVID-19 pandemic in Bangladesh: a GIS-based nationwide distribution. Sleep Med [Epub ahead of print] 26 Apr 2021. Available from: https:// doi.org/10.1016/j.sleep.2021.04.025

22 Hosen I, Pakpour AH, Sakib N, Hussain N, al Mamun F, Mamun MA. Knowledge and preventive behaviors regarding COVID-19 in Bangladesh: a nationwide distribution. PLOS One 2021; 16: e0251151.
23 Sakib N, Bhuiyan AKMl, Hossain S, al Mamun F, Hosen I, Abdullah AH, et al. Psychometric validation of the Bangla fear of COVID-19 scale: confirmatory factor analysis and Rasch analysis. Int J Ment Health Addict [Epub ahead of print] 11 May 2020. Available from: https://doi.org/10.1007/s11469-020-00289-x.

24 Ahorsu DK, Lin C-Y, Imani V, Saffari M, Griffiths MD, Pakpour AH. The Fear of COVID-19 Scale: development and initial validation. Int J Ment Health Addict [Epub ahead of print] 27 Mar 2020. Available from: https://doi.org/10.1007/ s11469-020-00270-8.

25 Lin C, Imani V, Majd NR, Ghasemi Z, Griffiths MD, Hamilton K, et al. Using an integrated social cognition model to predict COVID-19 preventive behaviours. $\mathrm{Br} J$ Health Psychol 2020; 25(4): 981-1005.

EXTRA EXTRA 